\title{
Ultrasonographic diagnosis of pancreatic diseases: this is all you need
}

\author{
Yoshiki Hirooka ${ }^{1}$
}

(C) The Japan Society of Ultrasonics in Medicine 2020

COVID-19 has continued to wreak havoc around the world since being declared a pandemic by the World Health Organization (WHO). According to a report from Johns Hopkins University updated at 1:00 p.m. on June 14, which is when I am writing this Editor's Note, the number of infected individuals is $7,766,952$ and the number of deaths is 429,736 . Creating an accurate roadmap for tackling COVID-19 appears to be difficult, which means we will continue to follow a path with an uncertain future. At the very least, I think we have no choice but to persevere through these uncertain times until a reliable medication/treatment and a safe vaccine are developed. It looks like a little more time is needed before the wisdom of the world defeats COVID-19.

Three review articles in a feature titled "Echocardiographic management of valvular heart disease", which was planned by Professor Yutaka Otsuji, were published in Vol. 47 No. 1 of the Journal of Medical Ultrasonics (JMU). Four very educational and interesting review articles in a feature called "Differential diagnosis and treatment of hepatocellular carcinoma: the role of ultrasound", which was planned by Professor Kazushi Numata, our Deputy Editor-in-Chief, were published in Vol. 47, No. 2. The current issue (Vol. 47, No. 3) contains the third feature titled "Ultrasonographic diagnosis of pancreatic diseases: this is all you need."

According to Projection of Cancer Mortality and Incidence in 2019 based on the latest statistics (CANCER STATISTICS IN JAPAN-2019) from the Foundation for Promotion of Cancer Research, the number of deaths due to pancreatic cancer ranks 4th (4th for men, 3rd for women) among all cancers. Excluding lung cancer, it ranks 3rd (3rd for men, 2nd for women). In the United States, the number of deaths due to pancreatic cancer is predicted to rank $2 \mathrm{nd}$ after lung cancer within the next 10 years if the current situation continues. According to the Clinical Practice Guidelines for Pancreatic Cancer 2019 published by the Japan Pancreas Society in 2019, the first step for diagnosis of pancreatic cancer is transabdominal ultrasonography, while the second step is endoscopic ultrasonography. In other words, ultrasonography is essential for diagnosis of pancreatic cancer. I am confident that you will fully appreciate the usefulness of medical ultrasound in the diagnosis and treatment of pancreatic cancer after reading the feature in the current issue. We know that detection of pancreatic cancer when the tumor is $\leq 10 \mathrm{~mm}$ in size leads to a better prognosis for the patient. Ultrasonography plays a key role in terms of accomplishing that goal. We are thinking about publishing a feature about this in a future issue. Some tumors require differentiation, and some involve inflammation as a precancerous state. Acute pancreatitis, chronic pancreatitis, autoimmune pancreatitis, and so forth are involved in pancreatic cancer, and we are thinking of publishing features about these, as well.

In addition to clinical ultrasound, JMU also contains a wide range of articles related to ultrasound in the field of engineering. In addition to organ-specific, clinical-based features, we plan to include more engineering-based topics in the future. The JMU Editorial Committee will continue to make efforts to create a journal that is useful for all researchers involved in medical ultrasound and ultrasound research. Thank you for your attention.

Publisher's Note Springer Nature remains neutral with regard to jurisdictional claims in published maps and institutional affiliations.
Yoshiki Hirooka

hirooka@med.nagoya-u.ac.jp

1 Department of Gastroenterology and Gastroenterological Oncology, Fujita Health University, Toyoake, Japan 